\title{
Editorial Special issue on information technology in China
}

\author{
Journal of Information Technology (2014) 29, 206-207. doi:10.1057/jit.2014.14
}

W e are pleased to introduce this special issue of the Journal of Information Technology on information technology (IT) in China. We use the term IT rather than information systems (IS), which seems to be more proper and conventional for an IS journal, because we intend to consider broad ranges of networked technologies, from IT infrastructure to IT applications, wherein IT refers to IS in a broad sense.

In the past quarter of a century, the rise of China has been a defining factor in global economic development. During this time, China has recorded an average annual GDP growth rate of about $10 \%$ and constructed a modern IT infrastructure across the whole country, supporting a 'digital economy.' IT has become an important part of business and social life in China and has brought with it some significant changes. A number of phenomena with regard to IT in China have gained considerable research interest. First, China faces challenges in the future growth of IT infrastructure. Institutional and legislative barriers exist in the convergence of the Internet, telecommunications, and media sectors. This convergence, which is a common practice in other countries, is driven by technological innovation and market demand. Second, China has the largest e-commerce market in the world. The IT service market of China is booming. Amazon, Google, and other major Internet firms have set up in China. However, most global companies cannot take a leading role in this country because of the failure to localize their global services. In addition, local competitors, such as Baidu and Huawei, have taken the innovative initiative to lead the local and global markets. Interesting lessons can be drawn from case studies on the business models of Chinese and foreign IT service providers in China. Third, China is catching up in IT, changing from an IT importer to main IT adopter, producer, and owner. China hosts international brands such as Alibaba and Huawei, and has an increasing role in international technological standard setting, such as the $3 \mathrm{G}$ mobile standard. Moreover, the government has promoted indigenous IT innovation. However, interesting issues exist in China's national system of IT innovation, namely, its successes, failures, and criticisms about a techno-nationalism that jeopardizes fair, open competition for private and foreign firms. Fourth, the central government has sought to use IT to promote socio-economic development, help reduce social divides, and create a more 'homogeneous society.' Therefore, the impact and challenge of the application of IT to health care, education, justice, agriculture, environmental protection, disaster alleviation etc. have gained considerable interest.

This special issue is devoted to offering a deep understanding on these IT challenges in China. It aims to draw out particular insights into the policy making, development, and application of IT in recent years that the world's most populated country have to offer from a variety of theoretical and methodological perspectives. Significant studies on IT in China have been reported. However, most people focus on IS usages at the micro organizational and individual levels, and this focus reflects generally well-established but rather bounded interest within IS society. This special issue intends to bring together studies on important recent IT developments and applications in China that have been relatively ignored in the literature.

This special issue attracted 46 submissions. After the screening process by the guest editorial team, 29 papers were sent out to undergo three rounds of double-blind full review by at least two reviewers. Finally, four papers were accepted, each offering specific areas that fit the objective of this special issue. With their study 'Research on IT in China: A call for greater contextualization,' Li, Gao, and Mao timely conducted a literature review on this topic, given that research on IT in China has gained significant attention in the international IS society and that substantial knowledge has been accumulated. This article considers the papers published in the period of 2000-2013 in 'Senior Scholars' 'Basket of Journals.' Among the approximately 3600 papers published in these eight journals during this period, 115 papers are identified as relevant to the topic of IT in China, which are systematically reviewed and analyzed. This research provides the international IS society a general understanding of IT research in China. Key research issues and trends are identified. First, in contrast to the findings of review works published in Chinese that covers many international IS journals and Chinese journals, the last four years of IT research in China have received far greater attention than the first decade of the new millennium. Moreover, Chinese scholars have formed an important scientific community in the international IS society. Second, unlike past reviews on IS research published in MISQ and Management Sciences, this paper categorizes the global distribution of authors and their research methods on IT in China. Five research streams are identified, namely, human behaviors in IS adoption and use; IS management; e-business; social media, digital collaborations and group support; industrial and societal issues in IS. Twenty-two research themes emerge from the five streams; these themes reveal the scholar interests in Chinese IS practices. There are some themes, such as electronic commerce, remaining understudied. Third, various research methods have been adopted to conduct IT research in China, the most popular of which are survey, case study, and laboratory experiments. Based on the review of past research, this paper proposes future directions in IT research in China. In each paper, the authors specifically focused on the effect on the Chinese and the approaches to contextualizing 
the research topic. The national- and social-level contexts, such as national culture, policies and regulation, and institutional and transitional characteristics, received the most attention. However, most of the research on the studied sample was not contextualized. Researchers adopted the existing constructs, arguments, or theories directly and only tested them in China, without striving further for contextualization. The authors argued that the Chinese IT phenomena could only be better understood in their local context, with the help of indigenous constructs. Contextual research on IT in China is becoming increasingly important at the national, organizational, and individual levels. Greater contributions can be made by conducting contextualized research and considering contextual elements in research design, including the selection of research phenomena and research methods for theory development.

In 'Network relations and boundary spanning: Understanding the evolution of e-ordering in the Chinese drug distribution industry,' Guo, Reimers, Xie, and Li discussed the application of e-ordering systems in the drug distribution industry, which is a kind of industrial inter-organizational IS neglected by scholars, in China. Drawing upon the network relation model and boundary spanning theory, the authors formulated a taxonomic framework for understanding interfirm network practices, which were used to explain long-term changes in drug distribution in China. It is found that practice in the field has evolved from traditional, socially embedded relations to IS-based, socially embedded relations; the centralized platform deployed by the government has been unable to establish a practice with arm's length relations. The developed theoretical framework can be used in studying inter-firm practices that explicitly incorporate the presence of interorganizational IS. The empirical findings offer helpful practical insights for facilitating collective efforts toward the innovative use of novel IT in the drug distribution industry in China and other similar contexts. The authors found that the Chinese context is particularly intriguing. Particularly, even in a context characterized by heavy-handed government intervention, industry practices may resist government intentions; such resistance does not result from coordinated collective action but emerges unexpectedly and possibly unintentionally from ongoing mutual adaptations of routines, strategies, and technologies. Thus, the authors argued that practice persistence in a particular context deserved academic scrutiny.

In 'Can customer satisfaction and dissatisfaction coexist? An issue of telecommunication service in China,' Chen, Lu, Gupta, and Qi conducted a quantitative research that added flavor to JIT, which usually publishes qualitative research. Relevant hypotheses were developed based on two-factor theory, negative bias theory, and cognition-effect-behavior framework. The sampling frame consists of customers of China Mobile with the largest group of subscribers in the world. The PLS analysis of the survey data collected from 679 respondents demonstrates that customer satisfaction and dissatisfaction can be managed simultaneously. This finding indicates the complex interrelationships between 11 telecommunications service attributes, functional perception, motivating perception, customer satisfaction, customer dissatisfaction, and customer loyalty constructs. The role of hygiene, motivation, and bivalent factors is identified. This research gives good effort in using Chinese data to test and extend Western theories. However, no Chinese constructs were developed, which could limit the depth for understanding the IT phenomenon in China. The authors found that some of the findings could be idiosyncratic to the contextual features of China Mobile but did not discuss this issue further.

In 'How do IT outsourcing vendors respond to shocks in client demand? A resource dependence perspective,' $\mathrm{Su}$, Mao, and Jarvenpaa focused on the dependence of outsourcing vendors in IS projects on their clients to reap gains and develop capabilities, putting vendors in a vulnerable position of receiving shocks from client demand. They examined how vendors mitigate the damage from demand shocks, which is a sidelined topic in IS literature. A multiple-case study on five pairs of relationships between Chinese vendors and their Japanese clients was conducted by drawing upon resource dependence theory, which considers two response strategies: bridging and buffering. The findings suggest that both bridging and buffering should be specified further based on their explorative and exploitative dimensions, and that the choice of a particular strategy depends on the power relation between the vendor and the client. Particularly, the vendor chooses bridging when the client is in a high power advantage. When the vendor also has high power, explorative bridging is adopted; otherwise, exploitative bridging is adopted. When the client is in a low power position, the vendor pursues explorative buffering. Exploitative buffering is a common response to demand shocks, independent of the dyadic power relation. This paper can go further to consider the influence of the Chinese context on the power positions of Chinese vendors and their outsourcing strategies.

In conclusion, we would like to thank all the authors and reviewers for their support in making this special issue possible. We believe that your effort is repaid by the premise of this special issue in reflecting extant knowledge and promoting the increased understanding and clearer view of future research on IT in China. Looking forward, we call for research that focuses on any stage of IT life cycle, from R\&D to production and usage, of diverse ITs in China, and addresses the opportunities and challenges of broad ranges of IT in China in various areas. The key aspects of IT should be linked to China's particular social, economic, cultural, political, and institutional context. Moreover, the Chinese context should be used to test and extend extant theories as well as to develop new theoretical insights into the specific phenomenon of IT in China.

\footnotetext{
Ping Gao ${ }^{1}$, Heejin Lee ${ }^{2}$, Kalle Lyytinen ${ }^{3}$, Kanliang Wang ${ }^{4}$ Special Issue Editors

${ }^{1}$ Institute for Development Policy and Management, University of Manchester, UK

${ }^{2}$ Graduate School of International Studies, Yonsei University, Korea ${ }^{3}$ Weatherhead School of Management, Case Western Reserve University, USA ${ }^{4}$ School of Business, Renmin University of China, China
} 